\title{
NASA EARTH EXCHANGE: NEXT GENERATION EARTH SCIENCE COLLABORATIVE
}

\author{
Ramakrishna Nemani \\ Ames Research Center, Moffett Field, CA
}

\begin{abstract}
The NASA Earth Exchange (NEX) is a collaboration platform for the Earth science community creating new ways for scientific interaction and knowledge sharing. NEX combines state-of-the-art supercomputing, Earth system modeling, workflow management, NASA remote sensing data feeds, and a social networking platform to deliver a complete work environment in which users can explore and analyze large datasets, run modeling codes, collaborate on new or existing projects, and quickly share results among the Earth science communities. The work environment provides NEX members with community supported modeling, analysis and visualization software in conjunction with datasets that are common to the Earth systems science domain. By providing data, software, and large-scale computing power together in a flexible framework, NEX reduces the need for duplicated efforts in downloading data, developing pre-processing software tools, and expanding local compute infrastructures-while accelerating fundamental research, development of new applications, and reducing project costs. The social networking platform provides a forum for NEX members to efficiently share datasets, results, algorithms, codes, and expertise with other members. Since all members' work environments reside on the collaborative platform, sharing may be done without the transfer of large volumes of data or the porting of complex codesmaking NEX an ideal platform for building upon and exchanging research, and fostering innovation.
\end{abstract}

